\title{
Search of new physics via $\eta$ rare decays
}

\section{Liping Gan*†}

University of North Carolina Wilmington, NC, USA

E-mail: ganl@uncw.edu

\section{Ashot Gasparian}

NC A\&T State University, NC, USA

E-mail: gasparan@jlab.org

\begin{abstract}
An experimental program to study $\eta$ rare decays with high precision and high statistics has been under development at Jefferson Lab with a $12 \mathrm{GeV}$ energy upgrade to test fundamental symmetries of QCD. The first suggested experiment is to measure $\eta \rightarrow \pi^{0} \gamma \gamma$ and $\eta \rightarrow \pi^{0} \pi^{0}$ channels simultaneously in Hall D. Three experimental techniques will be used to obtain the desired precision and statistics. This will include: (1) a $12 \mathrm{GeV}$ high intensity tagged photon beam for $\eta$ production through $\gamma p \rightarrow \eta p$ reaction; (2) tagging the $\eta$ 's by measuring the recoil $p$ with the GlueX detector; and (3) a high resolution, high granularity $\mathrm{PbWO}_{4}$ calorimeter to detect multiphotons from the $\eta$ decays.
\end{abstract}

6th International Workshop on Chiral Dynamics

July 6-10 2009

Bern, Switzerland

* Speaker.

${ }^{\dagger}$ This project is supported by US NSF grant 055524 . 


\section{Introduction}

In recent years, searching for new physics beyond the Standard Model has become a prime task for physicists. A good place to search for new physics is to test the fundamental symmetries, such as chiral symmetry, charge conjugation $\mathrm{C}$, parity $\mathrm{P}$, and time reversal $\mathrm{T}$, as well as $\mathrm{CP}$ and CPT, in the different interactions. As shown in Table 1, the measurements of branching ratios or upper limits for various rare and forbidden decays of $\eta$ will provide sensitive probes to test fundamental symmetries of QCD. We have been developing an experimental program to study $\eta$ rare decays, with the first attempt to measure $\eta \rightarrow \pi^{0} \gamma \gamma$ and $\eta \rightarrow \pi^{0} \pi^{0}$ simultaneously in Hall $\mathrm{D}$ at Jefferson Lab with $12 \mathrm{GeV}$ energy upgrade. This program can provide a great opportunity to understand symmetry structure of QCD and search for new physics beyond the Standard Model.

\begin{tabular}{|c|c|c|}
\hline Mode & Branching Ratio & Physics Highlight \\
\hline \hline$\pi^{0} \pi^{0}$ & $<3.5 \times 10^{-4}$ & $\mathrm{CP}, \mathrm{P}$ \\
\hline$\pi^{0} 2 \gamma$ & $(2.7 \pm 0.5) \times 10^{-4}$ & $\chi \mathrm{PTh}, \mathscr{O}\left(p^{6}\right)$ \\
\hline$\pi^{0} \gamma$ & $<9 \times 10^{-5}$ & $\mathrm{C}$ \\
\hline$\pi^{+} \pi^{-}$ & $<1.3 \times 10^{-5}$ & $\mathrm{CP}, \mathrm{P}$ \\
\hline$\pi^{0} \pi^{0} \gamma$ & $<5 \times 10^{-4}$ & $\mathrm{C}$ \\
\hline $3 \gamma$ & $<1.6 \times 10^{-5}$ & $\mathrm{C}$ \\
\hline$\pi^{0} \pi^{0} \pi^{0} \gamma$ & $<6 \times 10^{-5}$ & $\mathrm{C}$ \\
\hline$\pi^{0} e^{+} e^{-}$ & $<4 \times 10^{-5}$ & $\mathrm{C}$ \\
\hline $4 \pi^{0}$ & $<6.9 \times 10^{-7}$ & $\mathrm{CP}, \mathrm{P}$ \\
\hline
\end{tabular}

Table 1: Some interesting $\eta$ rare decay channels [1].

\section{The $\eta \rightarrow \pi^{0} \gamma \gamma$ decay}

The $\eta \rightarrow \pi^{0} \gamma \gamma$ decay channel has a very long and dramatic history (which is reviewed in reference [2]), and has attracted much attention from both theoretical and experimental points of view in the past four decades. In the chiral perturbation (ChPT) calculations, the tree level amplitudes vanish at both $\mathscr{O}\left(p^{2}\right)$ and $\mathscr{O}\left(p^{4}\right)$, and the first non-vanishing contribution comes from $\mathscr{O}\left(p^{4}\right)$ loop terms [3]. However, loops involving kaons are largely suppressed due to the kaon masses, while the pion loops are suppressed due to $\mathrm{G}$ parity. The first sizable contribution comes at $\mathscr{O}\left(p^{6}\right)$. Thus, this decay channel provides a unique probe for higher order corrections in ChPT. Because $\mathscr{O}\left(p^{6}\right)$ coefficients are not precisely determined, these effects cannot be calculated without model ambiguities. A recent article predicts that the two-photon invariant mass spectra have different shapes for different mechanisms in the process [4]. High statistical measurements of such spectra would be very desirable to resolve the model ambiguities.

About twenty experiments have been performed to measure this decay width since 1966. Despite considerable experimental effort, however, the first sufficiently sensitive result published by GAMS-2000 collaboration [5] in 1984 yields $\Gamma\left(\eta \rightarrow \pi^{0} \gamma \gamma\right)=0.84 \pm 0.18 \mathrm{eV}$, which is about two times larger than the average of the ChPT predictions as shown in Figure 1. In recent years, 


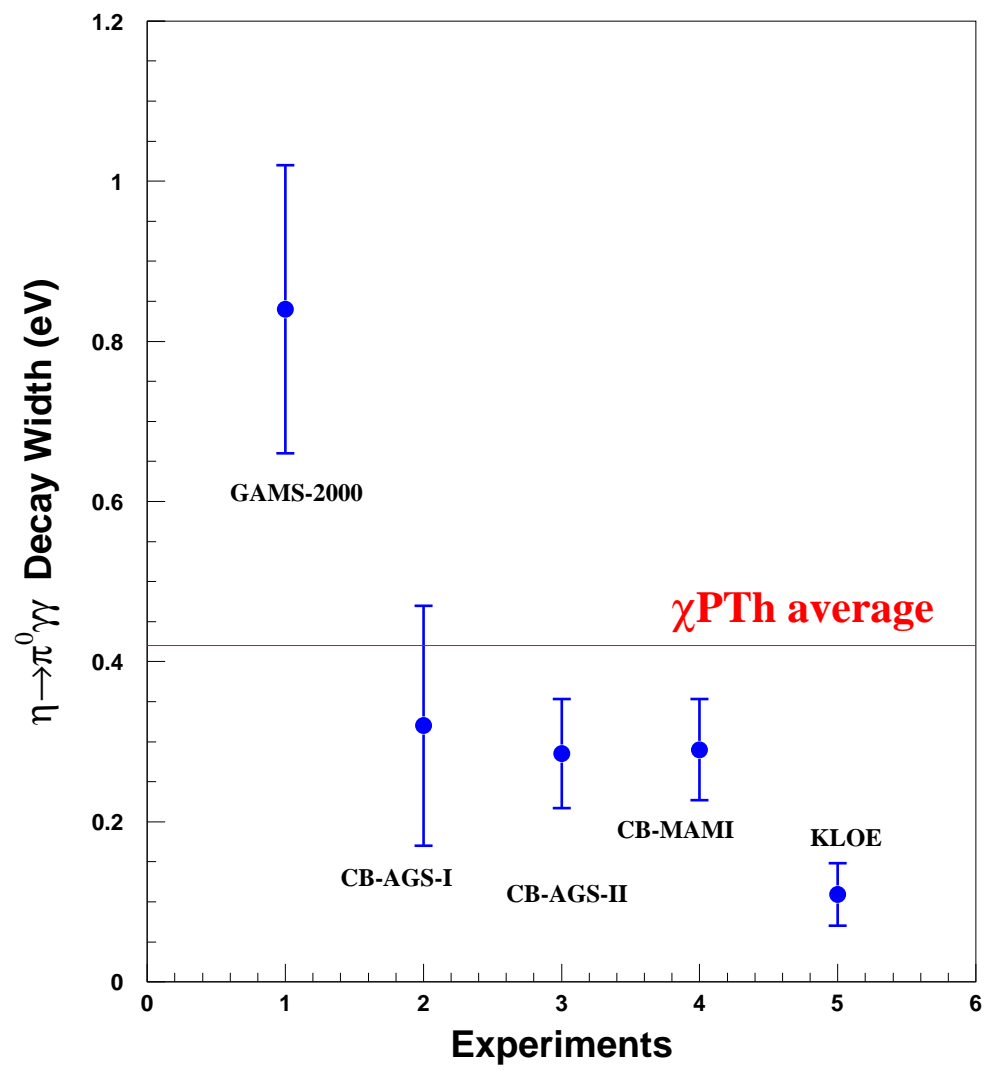

Figure 1: Experimental results on the decay width of $\eta \rightarrow \pi^{0} \gamma \gamma$. Point 1 is the GAMS-2000 result [5]. Point $2[6]$ and $3[7][8]$ are independent analysis results from Crystal Ball collaboration at AGS. Point 4 is the result from Crystal Ball collaboration at MAMI [9]. Point 5 is the result from KLOE collaboration [10]. The red solid line is the average result of ChPT predictions.

there have been several new experimental efforts from the Crystal Ball (at AGS and MAMI) and KLOE collaborations. These results are not consistent, and the values of $\Gamma\left(\eta \rightarrow \pi^{0} \gamma \gamma\right)$ vary from $0.32 \pm 0.15 \mathrm{eV}$ [6], $0.285 \pm 0.031 \pm 0.061 \mathrm{eV}$ [7][8], $0.290 \pm 0.059 \pm 0.022 \mathrm{eV}$ [9], to $0.109 \pm 0.035 \pm 0.018 \mathrm{eV}[10]$. These recent results also have a tendency to be below the average theoretical predictions shown in Figure 1, New experimental confirmation with higher precision is strongly desirable.

The major challenge in this measurement is to suppress the background from $\eta \rightarrow 3 \pi^{0}$. This decay channel has a branching ratio that is about three orders of magnitude larger than the desired $\eta \rightarrow \pi^{0} \gamma \gamma$ decay. Also, it imitates the signals mainly through two mechanisms [5]: (1) two soft photons from different pions left undetected, and (2) the merging of photons in the photon detector. Increasing the energy of $\eta$ mesons can dramatically improve the $\eta \rightarrow 3 \pi^{0}$ background suppression [2]. The low energy $(<1 \mathrm{GeV}) \eta$ means relatively high thresholds for $\gamma$-ray detection. As a result, the two softest $\gamma$ 's could easily escape undetected, and this is one of the two mechanisms 


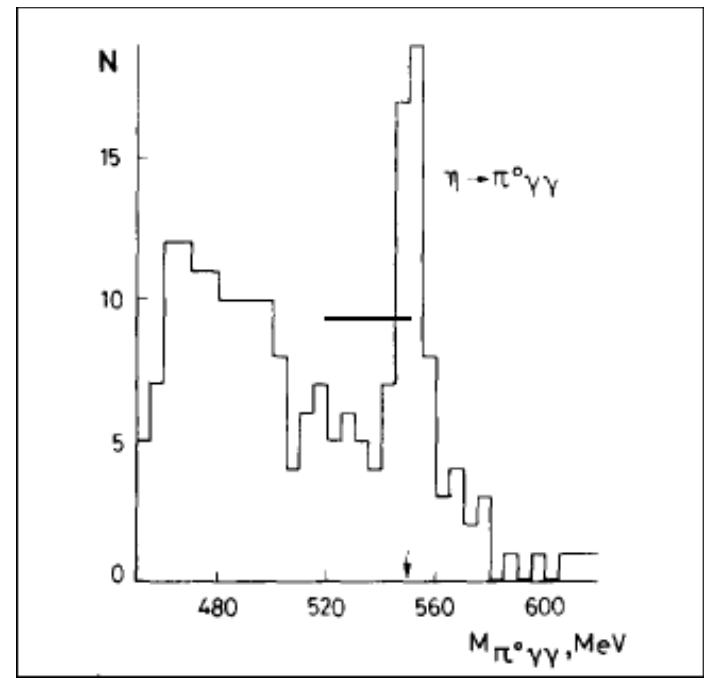

Figure 2: Invariant mass spectrum for the $\pi^{0} \gamma \gamma$ system measured by GAMS collaboration [5] with high energy $\eta$ production.

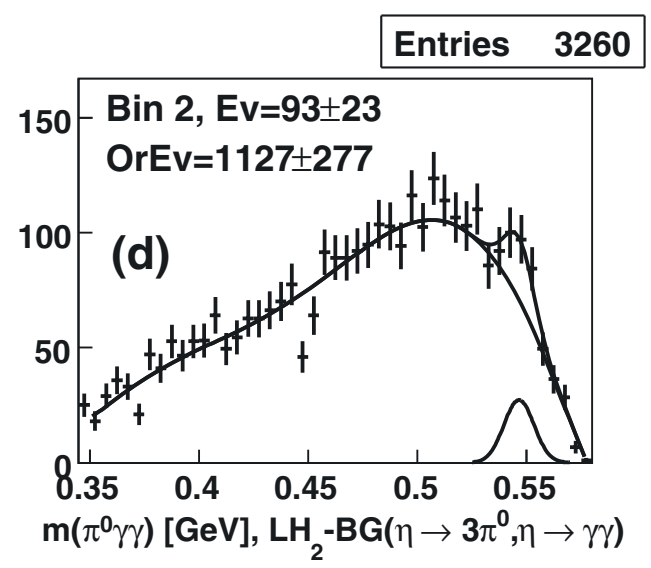

Figure 3: Invariant mass spectrum for the $\pi^{0} \gamma \gamma$ system measured by Crystal Ball collaboration at AGS [8] with the $\eta$ production near threshold.

for $\eta \rightarrow 3 \pi^{0}$ to mimic the $\eta \rightarrow \pi^{0} \gamma \gamma$ decay. With increasing $\eta$ energy, relative threshold for $\gamma$ detection gets lower, so that the $\eta \rightarrow 3 \pi^{0}$ background is reduced. On the other hand, higher decay photon energies will improve the invariant mass resolution of the detector, which will consequently further suppress the background significantly.

The advantage of using high energy $\eta$ production can be also seen by comparing the results from the existing experiments shown in Figure 1. One of the big differences between the GAMS experiment [5] and the recent experiments done by Crystal Ball and KLOE collaborations [6]-[10] is the energy of the $\eta$ mesons. In the GAMS experiment, the $\eta$ 's were produced by a high energy negative pion beam $(30 \mathrm{GeV})$ in the charge exchange reaction $\pi^{-} p \rightarrow \eta n$. The decay photons from $\eta$ decays were detected in the forward direction by a calorimeter consisting of a $48 \times 32$ matrix of lead glass Cerenkov counters. The background in this experiment is observed at the left of the $\eta$ peak in the invariant mass spectrum of the $\pi^{0} \gamma \gamma$ system. As shown in Figure 2, a narrow peak of $\eta \rightarrow \pi^{0} \gamma \gamma$ with a statistical significance exceeding seven standard deviations showed up on a smooth background. In the Crystal Ball and KLOE experiments, on the other hand, the $\eta$ 's were produced near the production threshold and the energy of decay $\gamma$ 's were less than $\sim 500 \mathrm{MeV}$ which were detected by a $4 \pi$ detector. The background from $\eta \rightarrow 3 \pi^{0}$ is typically peaked at the $\eta$ mass in the invariant mass spectrum of the $\pi^{0} \gamma \gamma$ system (see Figure 10 (a) in reference [7]) where the desired signature of $\pi^{0} \gamma \gamma$ is located. The overlap of the background and signal makes it difficult to have a clean event separation. Figure 3 shows the result from the Crystal Ball collaboration at AGS [8]. As one can see in Figure 2 and Figure 3, the background in the low energy $\eta$ production experiment is significantly worse than in the case of the high energy experiment.

As described above, the second important mechanism for the $\eta \rightarrow 3 \pi^{0}$ to mimic the $\eta \rightarrow$ $\pi^{0} \gamma \gamma$ decay is through overlapping photon clusters in the $\gamma$-detector. The high granularity devices, such as the calorimeter made out of $\mathrm{PbWO}_{4}$ crystals, which have Moliere radii $(\sim 2 \mathrm{~cm})$ about two times less than the typical radiator material (such as lead glass) used in an older generation 
calorimeter, would greatly help to discriminate merged photons in the detector by examining the energy deposition profile of the corresponding electromagnetic shower in the transverse direction. Our Monte Carlo simulation indicates that replacing the conventional lead glass calorimeter with the $\mathrm{PbWO}_{4}$ crystals will improve the suppression of the $\eta \rightarrow 3 \pi^{0}$ background due to overlapping showers by more than two orders of magnitude. The new generation of calorimeter techniques using $\mathrm{PbWO}_{4}$ crystals, where the energy and position resolutions are about two times better than lead glasses, also ensure much better angular and invariant mass resolutions than those used in the previous experiments.

Jefferson Lab's future $12 \mathrm{GeV}$ upgrade and planned high intensity, high energy photon tagging facility in Hall D will provide an unique opportunity to perform high precision and high statistics $\eta$ rare decay measurements. In past several years, we have been developing a possible new experiment to measure $\eta \rightarrow \pi^{0} \gamma \gamma$ decay in Hall D. The $\eta$ 's will be produced at a $30 \mathrm{~cm}$ liquid hydrogen target with a $12 \mathrm{GeV}$ tagged photon beam. A forward high resolution and high granularity $\mathrm{PbWO}_{4}$ crystal calorimeter, which was proposed for the Jlab $12 \mathrm{GeV}$ Primakoff experimental program [11], will detect multi-photons from the $\eta$ decays. The high energy $\eta$ production will not only help suppressing the $\eta \rightarrow 3 \pi^{0}$ background, but also increasing the detector's geometrical acceptance by boosting the photons to the forward direction. In addition, there are also some contributions coming from the non-resonance $\gamma p \rightarrow \pi^{0} \pi^{0} p$ or $\gamma p \rightarrow \pi^{0} \pi^{0} \pi^{0} p$ background. Tagging $\eta$ events by measuring the recoil protons with the GlueX detector will reduce significantly such non-resonance background.

\section{The $\eta \rightarrow \pi^{0} \pi^{0}$ decay}

Another interesting four-photon final state reaction is $\eta \rightarrow \pi^{0} \pi^{0}$ which is directly connected to $\mathrm{CP}$ and P symmetries. Since the discovery of a $0.2 \% \mathrm{CP}$ violation in 1964 came as a great surprise, the origin of this violation remains the most mysterious phenomenon in elementary particle physics. CP violation is incorporated into the Standard Model by means of complex coupling constants in the quark matrix and is controlled by a single parameter - the Kobayashi-Maskawa phase [12]. This violation shows up in family-changing interactions, while in family-conserving cases CP violation is observably small. The experimental verification of the second case is still very poor. The decay of $\eta \rightarrow \pi^{0} \pi^{0}$ is among four tests listed in the Review of Particle Physics [14] to study non-conventional CP violating effects. Since $\eta \rightarrow \pi^{0} \pi^{0}$ is a flavor-conserving interaction, the expected branching ratio in the Standard Model is small. A recent calculation indicates that $B R\left(\eta \rightarrow \pi^{0} \pi^{0}\right)$ should be less than $3 \times 10^{-17}$ [13]. The current experimental limit on the $\pi^{0} \pi^{0}$ decay of the $\eta$ is $B R\left(\eta \rightarrow \pi^{0} \pi^{0}\right)<3.5 \times 10^{-4}$ [1]. The discovery of a much larger decay rate would be a sign for the existence of a non-conventional CP violation mechanism. We can improve the upper limit of $\eta \rightarrow \pi^{0} \pi^{0}$ from the same data set of $\eta \rightarrow \pi^{0} \gamma \gamma$ measurement.

\section{Summary}

The Jefferson Lab $12 \mathrm{GeV}$ upgrade will provide a great opportunity for the precise measurements of various $\eta$ rare decays to test chiral, $\mathrm{P}, \mathrm{CP}$ and $\mathrm{C}$ symmetries, and search for new physics beyond the Standard Model. We have been in the process of developing an experimental program 
for $\eta$ rare decays, with the first attempt to measure $\eta \rightarrow \pi^{0} \gamma \gamma$ and $\eta \rightarrow \pi^{0} \pi^{0}$ simultaneously in Hall D to test the ChPT prediction, $\mathrm{P}$ and CP symmetries. We plan to apply three experimental techniques: (1) Jlab $12 \mathrm{GeV}$ high intensity tagged photon beam to produce $\eta$ mesons on a hydrogen target through $\gamma p \rightarrow \eta p$ reaction; (2) tagging the $\eta$ 's by measuring the recoil $p$ with the GlueX detector to reduce the non-resonance background from $\gamma p \rightarrow \pi^{0} \pi^{0} p$ or $\gamma p \rightarrow \pi^{0} \pi^{0} \pi^{0} p$ reactions; and (3) a forward high resolution, high granularity $\mathrm{PbWO}_{4}$ calorimeter to detect multi-photons from the $\eta$ decays to reduce the $\eta \rightarrow 3 \pi^{0}$ background.

\section{References}

[1] C. Amsler et al.(Particle Data Group), Phys. Lett., B667, 1 (2008).

[2] M.N. Achasov et al., Nucl. Phys. B600, 3 (2001).

[3] L. Ametller, J. Bijnens, A. Bramon and F. Cornet, Phys. Lett. B276, 185 (1992).

[4] E. Oset, J.R. Pelaez, L. Roca, Phys. Rev. D77, 073001 (2008).

[5] D. Alde et al., Yad. Fiz 40, 1447 (1984); D. Alde et al., Z. phys. C25, 225 (1984); L. G. Landsberg, phys. Rep., 128, 301 (1985).

[6] N. Knecht et al., phys. Lett., B589, 14 (2004).

[7] S. Prakhov et al., Phys. Rev. C72, 025201(2005).

[8] S. Prakhov et al., Phys. Rev. C78, 015206 (2008).

[9] S. Prakhov, Proceedings of the 11th International Conference on Meson-Nucleon Physics and the Structure of the Nucleon, Juelich, 2007, http://www.fz-juelich.de/ikp/menu2007/Program/ProgramSessions.shtml.

[10] B.Di Micco et al., Acta Phys. Slovaca 56, 403 (2006).

[11] CEBAF $12 \mathrm{GeV}$ upgrade white paper, Science Driving the $12 \mathrm{GeV}$ CEBAF Upgrade, 2001; Conceptual Design Report for the Science and Experimental Equipment for the $12 \mathrm{GeV}$ Upgrade of CEBAF, 2005.

[12] T.D. Lee and C.N. Yang, Phys, Rev. 104, 254 (1956); Z.K. Silagadze, Phys. At. Nucl. 60, 272 (1997).

[13] E. Shabalin, Eta Physics Handbook, Workshop on Eta Physics, Uppsala 2001; C. Jarlskog and E. Shabalin, Phys. Rev. D52, 248 (1995); C. Jarlskog and E. Shabalin, Phys. Rev. D52, 6327 (1995).

[14] Particle Data Book, Phys. Rev., D66, (2002). 